\title{
Multiple Constrained QoS Routing Algorithm Based On Genetic Algorithm In Overlay Network
}

\author{
JunWei GE \\ School of Communication and \\ Information Engineering \\ Chongqing University of Posts and \\ Telecommunications \\ ChongQing,China \\ gejw@cqupt.edu.cn
}

\author{
JinXin Deng \\ School of Communication and \\ Information Engineering \\ Chongqing University of Posts and \\ Telecommunications \\ ChongQing,China \\ dengjinxin0517@163.com
}

\author{
YiQiu Fang \\ College of Computer Science and \\ Technology \\ Chongqing University of Posts and \\ Telecommunications \\ ChongQing,China \\ fangyq@cqupt.edu.cn
}

\begin{abstract}
How to depend on different services provide different guarantee of QoS, and to ensure the effective use of network resources will become an important task in overlay network. In this paper, we consider constraints of network bandwidth, delay, loss rate, cost , and base on the resources of current global network, as well as user-specific QoS metric needs to choose the best QoS route based on genetic algorithm. Experiments show that the algorithm is feasible in overlay network routing,and it has the better global load balancing capabilities.
\end{abstract}

Keywords-Overlay Network; QoS; Genetic Algorithm; Routing Select; Load Balancing

\section{INTRODUCTION}

With the development and popularization of the application technology of the Internet,the current network has became uncontrollable, unmanageable, and can't solve the QoS, security, and other issues. Hence, Overlay network[1] appears in this condition which can provide more reliable, fault-tolerant and better service.Overlay network takes the users' QoS constraints needs into account to find a service path which has been proved a NP-Complete problem, we just can obtain the approximate optimal solution.

Heuristic algorithm,such as RBLCP algorithm [2],is the most commonly used methods to solve such problems.But the good or bad of the design of heuristic cost function is directly affect the choice of the optimal service path.The detecting routing algorithm put forward by Jaipal Singh is base on the greedy algorithm [3], which will broadcast routing information.Jun Hang put forward polynomial inspired algorithms [4] for polynomial solvable problem.This algorithm reduce the search space, and improve the running speed.Genetic algorithm is put forward by J.Hlland professor[5] which is a parallel algorithm, and is much more simple and effective to solve multi-constrained QoS routing problem.Thereby the GA will be used in this paper to enhance network load balancing.

\section{OVERLAY NETWORK MODEL AND MATHEMATICAL DESCRIPTION}

Overlay network can be expressed as shown in
Figure 1, which the $1,2, \ldots 10$ stand for cover node.There is a user in the area that is covered by 1,hence the 1 is the local cover node of user. 1 will find the next service node which behind the first service until the last service.So when users need to obtain an application service, you need to contact the local cover node, then the node compute the next cover node $\mathrm{X}$ which is next service of user need.Similarly, $\mathrm{X}$ compute the next service node $\mathrm{Y}$, until you find the the service destination node $\mathrm{N}$.

An overlay network can be described as a graph $\mathrm{G}(\mathrm{V}$, E),in which $V$ represents a single service node,$V=\{V 1$, $\mathrm{V} 2, \mathrm{~V} 3, \ldots . . \mathrm{VN}\}$, and $\mathrm{E}$ represents the coverage link, $\mathrm{E}=$ $\{\mathrm{e} 1, \mathrm{e} 2, \ldots . . \mathrm{eN}\}$.For example,there has a network application which compose of two ordered service,such as 1-5,we can find overlay network link like 1-4-5,or $1-2-3-4-5$ and so on.

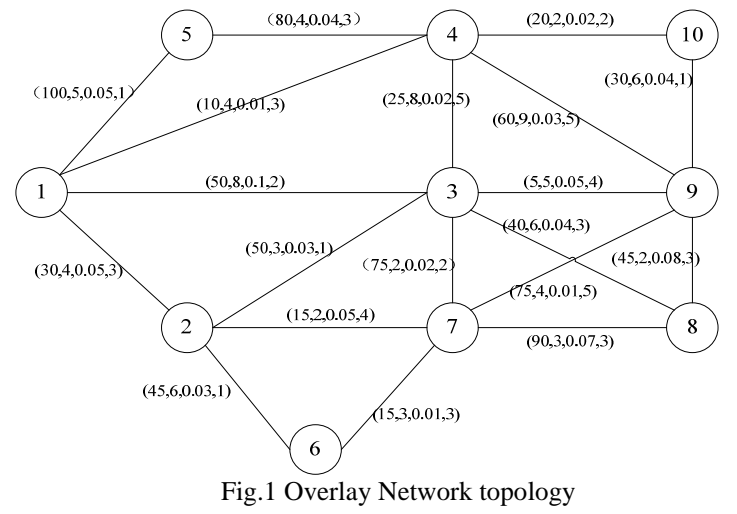

III. QOS ROUTING BASED ON GENETIC ALGORITHM

This article is based on the overlay network topology map $\mathrm{G}(\mathrm{V}, \mathrm{E})$.To satisfy a given constraint condition, finding an optimal service path from the source service node $\mathrm{s}$ to the destination service node $\mathrm{d}$ is the purpose of this article.Link bandwidth $\mathrm{B}$, the delay $\mathrm{D}$, the Cost $\mathrm{C}$, packet loss ratio $\mathrm{L}$, cover node processing capability $\mathrm{P}$ are the main factors that will be considered for $\mathrm{P}(\mathrm{s}, \mathrm{d})$ when QoS routing. The bandwidth, processing capability,the delay and the packet loss ratio from source service $s$ to destination d should satisfy the condition: 


$$
\begin{gathered}
\operatorname{Min} \mathrm{B}(\mathrm{a}, \mathrm{b}) \geq \mathrm{B} \\
(\mathrm{a}, \mathrm{b}) \in \mathrm{P}(\mathrm{s}, \mathrm{d}) \\
\underset{\mathrm{x} \in \mathrm{node}(\mathrm{s}, \mathrm{d})}{\mathrm{P}(\mathrm{X})} \geq \mathrm{P} \\
\sum_{(\mathrm{a}, \mathrm{b}) \in \mathrm{P}(\mathrm{s}, \mathrm{d})} \mathrm{D}(\mathrm{a}, \mathrm{b}) \leq \mathrm{D} \\
1-\prod_{(\mathrm{a}, \mathrm{b}) \in \mathrm{P}(\mathrm{s}, \mathrm{d})}(1-\mathrm{L}(\mathrm{a}, \mathrm{b})) \leq \mathrm{L}
\end{gathered}
$$

The cost from source service $\mathrm{s}$ to destination $\mathrm{d}$ should satisfy the condition:

$$
\operatorname{Cost}(\mathrm{s}, \mathrm{d})=\sum_{(\mathrm{a}, \mathrm{b}) \in \mathrm{P}(\mathrm{s}, \mathrm{d})} \mathrm{C}(\mathrm{s}, \mathrm{b})
$$

\section{A. Encoding method, and groups initialization}

In this paper,we use the depth-first search algorithm to find a path from source node $\mathrm{s}$ to the destination node $\mathrm{d}$. A single service node represents a gene, and a coverage service link is a chromosomes represented by the plurality of service nodes. This coding method will not coding and decoding in genetic operator.

We will do a pre-processing when population initialize.we will delete the link that it 's bandwidth dissatisfy the requirement of user and delete the cover node that it's capacity of computing dissatisfy the requirement of user,then we will get a new topology diagram.If it is not a connected diagram,and the path of service is not in a single connected diagram,the overlay network can't provide service.In this paper,we assume that is connected.

$B$. The design of the fitness function

The design of the fitness function is a key part of the genetic algorithm, and it directly impact the the speed of convergence of the algorithm, as well as the ability to find the optimal solution.In this paper,the fitness function as follows:

$$
\begin{gathered}
f(\mathrm{p})=C^{*}\left(\frac{\psi(\mathrm{P}(\mathrm{s}, \mathrm{d}))}{A^{*} \operatorname{Cost}(\mathrm{P}(\mathrm{s}, \mathrm{d}))+B^{*}\left(X^{*} \mathrm{D}(\mathrm{B})+Y^{*} \mathrm{D}(\mathrm{P})\right)}\right)^{1++ \text { Trgen }} \\
\quad \text { In this formula, } \psi(\mathrm{P}(\mathrm{s}, \mathrm{d})) \text { is a Penalty }
\end{gathered}
$$
function. When the delay or packet loss rate doesn't meet the requirement,its value will reduce.

$$
\begin{aligned}
& \psi(\mathrm{P}(\mathrm{s}, \mathrm{d}))=\psi_{d}(\mathrm{P}(\mathrm{s}, \mathrm{d})) \bullet \psi_{l}(\mathrm{P}(\mathrm{s}, \mathrm{d})) \\
& \psi_{d}(\mathrm{P}(\mathrm{s}, \mathrm{d}))=\left\{\begin{array}{l}
1, \quad \operatorname{delay}(\mathrm{P}(\mathrm{s}, \mathrm{d})) \leq \mathrm{D} \\
1-\frac{\operatorname{delay}(\mathrm{P}(\mathrm{s}, \mathrm{d}))-\mathrm{D}}{\operatorname{delay}(\mathrm{P}(\mathrm{s}, \mathrm{d}))}, \quad \operatorname{delay}(\mathrm{P}(\mathrm{s}, \mathrm{d}))>\mathrm{D}
\end{array}\right. \\
& \psi_{l}(\mathrm{P}(\mathrm{s}, \mathrm{d}))=\left\{\begin{array}{l}
1, \quad \operatorname{loss}(\mathrm{P}(\mathrm{s}, \mathrm{d})) \leq \mathrm{L} \\
1-\frac{\operatorname{loss}(\mathrm{P}(\mathrm{s}, \mathrm{d}))-\mathrm{L}}{\operatorname{loss}(\mathrm{P}(\mathrm{s}, \mathrm{d}))}, \quad \operatorname{loss}(\mathrm{P}(\mathrm{s}, \mathrm{d}))>\mathrm{L}
\end{array}\right. \\
& \mathrm{D}(\mathrm{B})=\sum_{(\mathrm{i}, \mathrm{j}) \in(\mathrm{s}, \mathrm{d})} \sqrt{\left(\mathrm{B}_{\mathrm{ij}}-\overline{\mathrm{B}}\right)^{2} / \mathrm{N}_{\mathrm{L}}} \quad \mathrm{D}(\mathrm{P})=\sum_{(\mathrm{i}, \mathrm{j})(\mathrm{s}, \mathrm{d})} \sqrt{\left(\mathrm{P}_{\mathrm{ij}}-\overline{\mathrm{P}}\right)^{2} / \mathrm{N}_{\mathrm{P}}} \\
& \operatorname{delay}(\mathrm{P}(\mathrm{s}, \mathrm{d}))=\sum_{(\mathrm{a}, \mathrm{b}) \in \mathrm{P}(\mathrm{s}, \mathrm{d})} \mathrm{D}(\mathrm{a}, \mathrm{b})
\end{aligned}
$$

$$
\operatorname{loss}(\mathrm{P}(\mathrm{s}, \mathrm{d}))=1-\prod_{(\mathrm{a}, \mathrm{b}) \in \mathrm{P}(\mathrm{s}, \mathrm{d})}(1-\mathrm{L}(\mathrm{a}, \mathrm{b}))
$$

In Fitness formula, $\mathrm{C}$ is a constant.The coefficients $\mathrm{A}$, $\mathrm{B}, \mathrm{X}, \mathrm{Y}$ are to balance the role of each parameter.If a parameter of denominator is too large,then the influence of the other parameters will be ignored. $\mathrm{D}(\mathrm{B})$ and $\mathrm{D}(\mathrm{P})$ stand for load balance of a link and nodes from node $s$ to node d.The smaller the value of $\mathrm{D}(\mathrm{B})$ and $\mathrm{D}(\mathrm{P})$ are,the more balance the globe load is.

$$
\begin{gathered}
X=\frac{\mathrm{D}(\mathrm{P})}{\mathrm{D}(\mathrm{B})+\mathrm{D}(\mathrm{P})} \\
Y=\frac{\mathrm{D}(\mathrm{B})}{\mathrm{D}(\mathrm{B})+\mathrm{D}(\mathrm{P})} \\
A=\frac{X^{*} \mathrm{D}(\mathrm{B})+Y^{*} \mathrm{D}(\mathrm{P})}{\operatorname{Cost}(\mathrm{P}(\mathrm{s}, \mathrm{d}))+X^{*} \mathrm{D}(\mathrm{B})+Y^{*} \mathrm{D}(\mathrm{P})} \\
B=\frac{\operatorname{Cost}(\mathrm{P}(\mathrm{s}, \mathrm{d}))}{\operatorname{Cost}(\mathrm{P}(\mathrm{s}, \mathrm{d}))+X^{*} \mathrm{D}(\mathrm{B})+Y^{*} \mathrm{D}(\mathrm{P})}
\end{gathered}
$$

The exponential is used to pull the differences between individuals in the latter of the selection to improve the convergence speed, and to find the optimal solution.

\section{Genetic operation}

1)Select the individual.In this paper, the roulette wheel selection method will be used, which is a simple choice.First of all,we use probability of individual fitness to determine the possibility of their inheritance.Then we will obtain the cumulative probability for each individual.Finally,it will Generating a random number from 0 to 1 as a selection pointer to determine which the individual will be selected.The greater the fitness of individual is,the greater the possibility of be selected is.

2)Cross the individuals.First, we select the parent link Px and Py to participate in the crossover operation ,and randomly select a service node as a hybrid node that are the same service in Px and Py.Secondly, all nodes after the crossover point in Px and Py will be changed,then we will get the new individual. we will Removed the nodes from the same node of first to the node which is ahead of the next same node in new individuals.If it does not meet the service order or lack of service after removing,we will not delete the loop. If there doesn't exist link connection between adjacent node, we will use the A-Star[6] algorithm to find the connection between them.

3)Mutate the individuals.First of all,we will randomly select a individual from the current population,which is based on the mutation probability,Then randomly select a node $x$ which is not service node from the individual.Secondly,we will remove the node x.If there is no connection between the node $\mathrm{m}$ which is before node $\mathrm{x}$ and node $\mathrm{n}$ which is behind the node $\mathrm{x}$ after remove the node $\mathrm{x}$,we will use A-Star algorithm whose parameter of link is delay or bandwidth or cost to find a way from service node $m$ to service node $n$. Finally, we will use this path to replace the original path.if there is a loop after the path is replaced, we will remove it refer to the method 
which is used in crossover operation.

\section{EXPERIMENT SIMULATION AND ANALYSIS}

In the simulation process, we only verify the feasibility of the proposed algorithm and its better load balance ability.So assume that each overlay node only to provide a service, and different with other nodes. This article uses a randomly generated 10-node network topology in which the processing capability of the coverage nodes is between 1 and 10, each link bandwidth is between 1 and 10,each link delay is between 10 and 100, the loss rate is between 0.01 and 0.1 .The cost is between 1 and 5.User requirs the bandwidth is equal to 2, Process capacity is equal to 1 ,delay less than $200 \mathrm{~ms}$, the loss rate less than 0.2 ,we will use the Figure 1 to simulate. The value of the link represent delay,bandwidth,loss rate,cost.And the process capacity from node 1 to node 10 is $10,8,6,4,6,4,9,3,4,7$.

1)Verify the feasibility of the algorithm. To select Population size ,popsize is equal to 5 , the genetic iteration number, maxgen is equal to 20 , and the source node is 1,destination node is 10.Then the cost of the link of the best path of each generation ,and equilibrium degree curve of process capacity of covering node and the link bandwidth as follows:

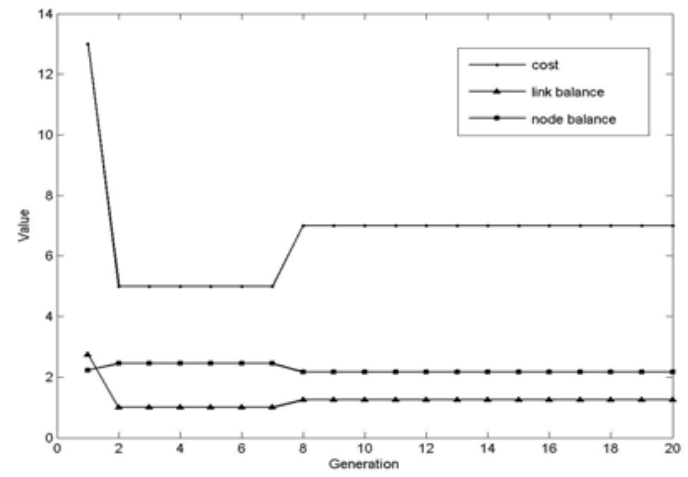

Fig.2 The change of cost and link balance and node balance in GA

In Figure 2, with the increase of the number of iterations of the genetic algorithm, we can see that the optimal routing link is changing.its cost, the link balance, node balance are changing, which indicate that the genetic algorithm in the overlay network is feasible.At the same time,the best path is 1-3-9-10 whose link delay less than require delay,and the link loss rate less than require loss rate.So we can know using the the improved genetic algorithm to find a path which meet the requirements including delay,bandwidth,process capacity,loss rate is feasible.

2) Compare with RBLCP algorithm,We can know the 8th iterations of genetic algorithm can find the best path 1-3-9-10 as follows:
TABLE I. COMPARISON OF ALGORITHMS

\begin{tabular}{|c|c|c|c|c|}
\hline Algorithm & Best path & Fitness & Cost & Delay \\
\hline $\begin{array}{c}\text { Improved } \\
\text { GA }\end{array}$ & $1-3-9-10$ & 57 & 7 & 85 \\
\hline RBCLP & $1-4-9-10$ & 27 & 9 & 100 \\
\hline
\end{tabular}

TABLE II. COMPARISON OF ALGORITHMS

\begin{tabular}{|c|c|c|c|c|}
\hline Algorithm & Best path & Loss rate & $\begin{array}{c}\text { Link } \\
\text { Balance }\end{array}$ & $\begin{array}{c}\text { Node } \\
\text { Balance }\end{array}$ \\
\hline $\begin{array}{c}\text { Improved } \\
\text { GA }\end{array}$ & $1-3-9-10$ & 0.179 & 1.247 & 2.165 \\
\hline RBCLP & $1-4-9-10$ & 0.078 & 2.054 & 2.487 \\
\hline
\end{tabular}

We can see in Table 1 and Table 2,the loss rate greater than RBCLP algorithm, but its value is less than 0.2 ,which meet the requirement of loss rate.Apart from that,the path seeked by the improved genetic algorithm in terms of cost, delay less than RBCLP algorithm, and the improved genetic algorithm are far RBCLP algorithm in terms of global network load balance.

\section{CONCLUSION}

This paper analyzes the mathematical model of the overlay network,in which consider network bandwidth, delay, packet loss rate, cost, node processing capacity.On the basis of this model, based on the improved genetic algorithm proposed in this paper, and the comprehensive utilization of network resources,we simulate it through experiments. The results show that the improved genetic algorithm in the overlay network routing is feasible and has a better global load balancing capabilities.

\section{REFERENCES}

[1] B.Y.Zhao, L.Huang, J.String, S.C.Rhea, A.D.Joseph, J.D.Kubiatowic, "Tapestry:A resilient globle-scale overlay for service deployment",IEEE Journal on Selected Areas in Communications,2004.1,22(1),pp.41-53.

[2] Ying Bi-Di,Chen Hui-Fang,Zhu Sun-Bin,Qiu Pei-Liang. RBLCP: Improved QoS-aware routing algorithm for overlay networks.Journal of Zhejiang University, 2007, 41(4):646-649(in Chinese).

[3] Jaipal Singh.Multicast QoS routing using Collaborative Path Exploration.2010 24th IEEE International Conference on Advanced Information Networking and Application,2010(8): Page(s):120-125.

[4] Jun Huang,Tanaka Y.Qos routing algorithms using fully polynomial time approximation scheme.2011 IEEE 19th international Workshop on Quality of Service(IWQOS),2011, Page(s):1-3.

[5] Holland J H. Concerning effcient adaptive systems[J].Yovits M C.Self Organizing Systems,1962: 215-230.

[6] Zhanqi DONG,Mingxia LI.A Routing Method of Ad Hoc Networks Based on A-star Algorithm.2009 International Conference on Networks Security,Wireless Communications and Trusted Computing.2009,Page(s):623-626. 